\title{
LMI Formulation for Static Output Feedback Design of Discrete-Time Switched Systems
}

\author{
Selma Ben Attia, Salah Salhi, and Mekki Ksouri \\ Electrical Branch, National Engineering School of Tunis (ENIT), University EL Manar, BP-37, le Belvdere, 1002 Tunis, Tunisia \\ Correspondence should be addressed to Selma Ben Attia, benattiaselma@yahoo.fr
}

Received 12 January 2009; Revised 9 September 2009; Accepted 7 October 2009

Recommended by Pablo Iglesias

\begin{abstract}
This paper concerns static output feedback design of discrete-time linear switched system using switched Lyapunov functions (SLFs). A new characterization of stability for the switched system under arbitrary switching is first given together with $\gamma$ performance evaluation. The various conditions are given through a family of LMIs (Linear Matrix Inequalities) parameterized by a scalar variable which offers an additional degree of freedom, enabling, at the expense of a relatively small degree of complexity in the numerical treatment (one line search), to provide better results compared to previous one. The control is defined as a switched static output feedback which guarantees stability and $\gamma$-performance for the closed-loop system. A numerical example is presented to illustrate the effectiveness of the proposed conditions.
\end{abstract}

Copyright (C) 2009 Selma Ben Attia et al. This is an open access article distributed under the Creative Commons Attribution License, which permits unrestricted use, distribution, and reproduction in any medium, provided the original work is properly cited.

\section{Introduction}

As computers, digital networks, and embedded systems become ubiquitous and increasingly complex, one needs to understand the coupling between logic-based components and continuous physical systems. This prompted a shift in the standard control paradigm in which dynamical systems were typically described by differential or difference equations to allow the modeling, analysis, and design of systems that combine continuous dynamics with discrete logic. This paradigm is called hybrid systems [1]. The modeled process is then described by several functioning modes and the switching between the modes is controlled by the evolution of internal variables of the system or by an external law which is not mastered. When the representation of functioning modes is made by means of linear time invariant models, the obtained model belongs to the class of linear switching systems. Switched linear systems are an important class of Hybrid Dynamical Systems (HDSs). They are dynamical systems containing simultaneously mixtures of logic and continuous dynamics [2,3]. A switched system is then represented by a set of continuous-time or discrete-time subsystems and rules that orchestrate the switching among them. The motivation for studying switched systems is from the fact that many practical systems are required to describe their hybrid behavior which may depend on various environmental factors and also because the modeling of many complex systems is only possible through the combination of the classical continuous laws of control with a logical discrete technique [4]. Another source of motivation for studying switched systems comes from the rapidly developing area of switching control. Control techniques based on switching between different controllers have been applied extensively in recent years, particularly in the adaptive context, where they have been shown to achieve stability and improve transient response [1]. The importance of such control methods also stems in part from the existence of systems that cannot by asymptotically stabilized by a single continuous feedback control law. In practice, switched systems can be applied to various modeling and control problems present in robotics, automotive systems, process control, power systems, air traffic control, switching power converters, and many other fields which include the modeling of communication networks, networked control systems, the modeling of biochemical reactions, the control of nonlinear systems that cannot be stabilized by continuous control laws, and the control of systems with large uncertainty using logicbased supervisors $[1,5]$. In recent years, particular efforts of 
researches have received an increasing interest and a growing attention in the study of the stability analysis and control design for switched systems [6-10]. We address the stability issues and control synthesis for discrete switched systems under arbitrary switching sequences. In fact, much efforts have been devoted to establishing analysis tools such as multiple Lyapunov functions (MLFs), piecewise Lyapunov function (PLF), and switched Lyapunov function (SLF) [1113]. The approach used in this note is based in the existence of a particular quadratic Lyapunov function making full use of switching nature of the switched system, specially it has the same switching signals as the switched system. It is called SLF (Switched Lyapunov Function) which is a switchingsequence dependent Lyapunov function. It is proved in this note that with the use of switched Lyapunov function less conservative results can be obtained $[14,15]$. Then, the results proposed in this work can be considered as a tradeoff between highly conservative results (those using a single quadratic Lyapunov function) and less conservative results. This paper intends to study the problem of stabilization of discrete switched systems by static output feedback. The main motivation for studying SOF controller comes partly from the fact that static output feedback (SOF) control is very useful and more realistic, since it can be easily implemented with low cost [16-18]. A new LMI formulation that uses a scalar variable is proposed, which makes it useful and interesting for design problems. It is shown that the proposed method can work successfully in situations where the existing methods fail [14]. This note is organized as follows. In Section 2, we introduce the problem formulation and we give some preliminaries. Section 3 is devoted to stability characterization and $\gamma$-performance analysis of switched systems by mean of a switched quadratic Lyapunov function. In Section 4, new sufficient LMI conditions are deduced to obtain stabilizing SOF controller gains. Then, the method is extended to SOF controller with $\gamma$-performance. A numerical evaluation is provided in Section 5. Finally, the paper is concluded in Section 6.

Notation. The notations used throughout the paper are standard. The relation $A>B(A<B)$ means that the matrix $A-B$ is positive (negative) definite. The matrix I denotes identity matrix of appropriate dimension. ${ }^{*}$ is used for the blocks induced by symmetry. $N$ is the number of subsystems. Conv \{\} stands for convex combination. $\xi$ denotes the set of indexes.

\section{Problem Formulation and Preliminaries}

Consider a linear switched system in the discrete time domain described by the following state equation:

$$
\begin{gathered}
x(k+1)=A_{i} x(k)+B_{i} u(k), \\
y(k)=C_{i} x(k),
\end{gathered}
$$

where $x(k) \in R^{n}$ is the state vector of the system at time $k, u(k) \in R^{m}$ is the control input, and $y(k) \in R^{p}$ is the measured output. The switching sequence is defined through a switching vector $\xi(k)=\left[\xi_{1}(k), \ldots, \xi_{N}(k)\right]^{T}$ whose components take the value 0 or 1 and satisfy

$$
\sum_{i=1}^{N} \xi_{i}(k)=1
$$

which means that at each instant $k$ only one component $\xi_{i}(k)$ is nonnull. At each time instance $k$, the dynamic matrix of the discrete linear system jumps on one of the elements of the set $\left[A_{1}, A_{2}, \ldots, A_{N}\right]$. In fact the formulation (1) is a particular case of the classical polytopic LPV one and, as such, the usual definitions for stability are available [12].

As $[8,9,18]$, the following assumptions are made:

$H_{1}$ : the switching rule $\xi(k)$ is not known a priori, but its value is real-time available,

$H_{2}$ : the matrices $C_{i}$ are of full row rank,

$H_{3}$ : for each mode, the pairs $\left(A_{i}, B_{i}\right)$ and $\left(A_{i}, C_{i}\right)$ are stabilizable and detectable, respectively.

We present now two useful lemmas used in the proofs later in the paper.

Lemma 1 (Projection Lemma). Given a symmetric matrix $\Psi \in I R^{m \times m}$ and two matrices $P, Q$ of column dimension $m$ there exists $X$ such that the following LMI holds:

$$
\psi+P^{T} X^{T} Q+Q^{T} X P<0,
$$

if and only if the projection inequalities with respect to $X$ are satisfied:

$$
N_{P}^{T} \psi N<0, \quad N_{Q} \psi N_{Q}^{T}<0,
$$

where $N_{p}$ and $N_{Q}$ denote arbitrary bases of the nullspaces of $P$ and $Q$, respectively.

Proof. See [19].

Lemma 2. Let $\Phi$ a symmetric matrix and $N$, $J$ matrices of appropriate dimensions. The following statements are equivalent:

(i) $\Phi<0$ and $\Phi+N J^{T}+J N^{T}<0$,

(ii) there exists a matrix $G$ such that

$$
\left[\begin{array}{cc}
\Phi & J+N G \\
J^{T}+G^{T} N^{T} & -G-G^{T}
\end{array}\right]<0 .
$$

Proof. Straightforward [19], apply the projection lemma on

$$
\begin{gathered}
{\left[\begin{array}{cc}
\Phi & J+N G \\
J^{T}+G^{T} N^{T} & -G-G^{T}
\end{array}\right]} \\
\quad=\left[\begin{array}{ll}
\Phi & J \\
J^{T} & 0
\end{array}\right]+\left[\begin{array}{c}
N \\
-I
\end{array}\right] G\left[\begin{array}{ll}
0 & I
\end{array}\right]+\left[\begin{array}{l}
0 \\
I
\end{array}\right] G^{T}\left[\begin{array}{ll}
N^{T} & -I
\end{array}\right] .
\end{gathered}
$$


In the following sections, the problems of stability analysis with $\gamma$-performance and static output feedback control for linear switched systems are addressed. Improved LMI sufficient condition for asymptotic stability of the origin equilibrium of (1) is provided. This condition is extended to give improved LMI for switched SOF control law.

\section{Stability Characterization and $\gamma$-Performance Analysis}

3.1. Stability Characterization. To check stability of the switched system (1) let the switched Lyapunov function be defined as [14]

$$
V(k, x(k))=x(k)^{T} P(\xi(k)) x(k)=x(k)^{T}\left(\sum_{i=1}^{N} \xi_{i}(k) P_{i}\right) x(k),
$$

where $P_{1}, \ldots, P_{N}$ are symmetric positive definite matrices. If such a positive definite Lyapunov function exists and its increment $\Delta V\left(k, x_{k}\right)=V\left(k+1, x_{k+1}\right)-V\left(k, x_{k}\right)$ is negative definite along the solution of (1), then the origin of the switched system is globally asymptotically stable according to the following.

Theorem 1 (see [20]). The origin equilibrium point of

$$
x(k+1)=f_{k}(x(k))
$$

is globally uniformly asymptotically stable if there exists a function $V: Z^{+} \times R^{n} \rightarrow R$ such that

(i) $V(k, x)$ is a positive definite function, decreasing and radially unbounded and $V(k, 0)=0$ for all $k \geq 0$;

(ii) $\Delta V\left(k, x_{k}\right)=V\left(k+1, x_{k+1}\right)-V\left(k, x_{k}\right)$ is negative definite along the solution of (8).

The Lyapunov function (7) is a positive definite function, decreasing and radially unbounded since

$$
\beta_{1}\left\|x_{k}\right\|^{2} \leq V\left(k, x_{k}\right)=x_{k}^{T}\left(\sum_{i=1}^{N} \xi_{i}(k) P_{i}\right) x_{k} \leq \beta_{2}\left\|x_{k}\right\|^{2},
$$

$\beta_{1}=\min _{i \in \xi} \lambda_{\min }\left(P_{i}\right), \beta_{2}=\max _{i \in \xi} \lambda_{\max }\left(P_{i}\right), V(k, 0)=0$, for all $k \geq 0$.

Theorem 2. The following statements are equivalent.

(i) There exists a Lyapunov function of the form (7) whose difference is negative definite, implying asymptotic stability of (1).

(ii) There exist $N$ symmetric matrices $P_{1}, \ldots, P_{N}$ satisfying

$$
\left[\begin{array}{cc}
-P_{i} & A_{i}^{T} P_{j} \\
P_{j} A_{i} & -P_{j}
\end{array}\right]<0, \quad \forall(i, j) \in \xi \times \xi .
$$

(iii) There exist $N$ symmetric matrices $S_{1}, \ldots, S_{N}$ and $N$ matrices $G_{1}, \ldots, G_{N}$ satisfying

$$
\left[\begin{array}{cc}
-G_{i}-G_{i}^{T}+S_{i} & G_{i}^{T} A_{i}^{T} \\
A_{i} G_{i} & -S_{j}
\end{array}\right]<0, \quad \forall(i, j) \in \xi \times \xi .
$$

(iv) There exist $N$ symmetric matrices $S_{1}, \ldots, S_{N}$ and $N$ matrices $G_{1}, \ldots, G_{N}$ satisfying

$$
\left[\begin{array}{cc}
\alpha_{i}^{2} S_{i}-S_{j} & A_{i} G_{i}+\alpha_{i} G_{i}-\alpha_{i} S_{i} \\
* & S_{i}-G_{i}-G_{i}^{T}
\end{array}\right]<0, \quad \forall(i, j) \in \xi \times \xi,
$$

where $\alpha_{i}$ are scalars belonging to ] - 1, 1[.

The Lyapunov function is given by $V(k, x(k))=$ $x(k)^{T}\left(\sum_{i=1}^{N} \xi_{i}(k) P_{i}\right) x(k)=x(k)^{T}\left(\sum_{i=1}^{N} \xi_{i}(k) S_{i}^{-1}\right) x(k)$.

Proof. (1) (i) $\Leftrightarrow$ (ii) $\Leftrightarrow$ (iii) see $[14,15]$

(2) $(\mathrm{i}) \Leftrightarrow($ iv). Premultiplying and postmultiplying (11) by $\operatorname{diag}\left\{P_{i}^{-1}, P_{j}^{-1}\right\}=\operatorname{diag}\left\{S_{i}, S_{j}\right\}$ one gets

$$
\left[\begin{array}{cc}
-S_{i} & S_{i} A_{i}^{T} \\
* & -S_{j}
\end{array}\right]<0
$$

which can be written as

$$
\begin{aligned}
{\left[\begin{array}{cc}
-S_{i} & S_{i} A_{i}^{T} \\
* & -S_{j}
\end{array}\right]=} & \underbrace{\left[\begin{array}{cc}
-S_{i} & -\alpha_{i} S_{i} \\
-\alpha_{i} S_{i} & -S_{j}
\end{array}\right]}_{\Phi}+\left[\begin{array}{c}
S_{i} \\
0
\end{array}\right]\left[\begin{array}{ll}
0 & A_{i}^{T}+\alpha_{i} I
\end{array}\right] \\
& +\left[\begin{array}{c}
0 \\
A_{i}+\alpha_{i} I
\end{array}\right]\left[\begin{array}{ll}
S_{i} & 0
\end{array}\right]<0,
\end{aligned}
$$

$\Phi<0$, for all $\alpha_{i} \backslash-1<\alpha_{i}<1$. Applying Lemma 1 , this is equivalent to the existence of matrices $G_{i}$ such that

$$
\left[\begin{array}{ccc}
-S_{i} & -\alpha_{i} S_{i} & S_{i} \\
-\alpha_{i} S_{i} & -S_{j} & A_{i}^{T} G_{i}+\alpha_{i} G_{i} \\
S_{i} & G_{i}^{T} A_{i}^{T}+\alpha_{i} G_{i}^{T} & -G_{i}-G_{i}^{T}
\end{array}\right]<0,
$$

and by the Schur complement with the block 1, 1 as a pivot, one directly gets (12).

Remarks. (i) For $\alpha_{i}=0$, the results developed in [14] are recovered.

(ii) Since $\left.\alpha_{i} \in\right]-1,1[$, the LMI condition (12) is of infinite dimension which may be undertaken by an online search over the scalar $\alpha_{i}$.

For the following section, let the switched system be

$$
\begin{aligned}
x(k+1) & =\sum_{i=1}^{N} \xi_{i}(k)\left(A_{i} x(k)+B_{i}^{\omega} w(k)\right), \\
z(k) & =\sum_{i=1}^{N} \xi_{i}(k)\left(C_{i}^{z} x(k)+D_{i}^{z w} w(k)\right),
\end{aligned}
$$

where $x(k) \in I R^{n}$ is the state vector of the system, $x(0)=$ $0, w(k) \in I R^{q}$ is the disturbance, and $z(k) \in I R^{p}$ is an output vector. $\xi_{i}(k)$ is a switching rule defined previously. Hence, the matrices $\left(A_{i}, B_{i}^{w}, C_{i}^{z}, D_{i}^{z w}\right)$ are allowed to take values, at an arbitrary discrete time instant, in the finite set $\left\{\left(A_{1}, B_{1}^{w}, C_{1}^{z}, D_{1}^{z w}\right), \ldots,\left(A_{N}, B_{N}^{w}, C_{N}^{z}, D_{N}^{z w}\right)\right\}$. 
3.2. Improved LMI for $\gamma$-Performance Analysis. The switched system (16) is said to have $\gamma$-performance [21] if it is asymptotically stable and

$$
\gamma^{-1} \sum_{k=0}^{\infty} z(k)^{2}<\gamma \sum_{k=0}^{\infty} w(k)^{2}, \quad \forall \sum_{k=0}^{\infty} w(k)^{2}>0 .
$$

Theorem 3. The following statements are equivalent.

(i) The switched system is asymptotically stable with $\gamma$-performance, if there exist symmetric matrices $\left\{P_{i}>0\right\}_{i=1}^{N}$ such that

$$
\left[\begin{array}{cccc}
-P_{i} & * & * & * \\
0 & -\gamma^{2} I & * & * \\
A_{i} & B_{i}^{w} & -P_{j} & * \\
C_{i}^{z} & D_{i}^{z w} & 0 & -I
\end{array}\right]<0
$$

(ii) The switched system is asymptotically stable with $\gamma$-performance, if there exist symmetric matrices $\left\{S_{i}>0\right\}_{i=1}^{N}$, a matrix $G_{i}$, and a scalar variable $\alpha_{i} \in$ ] $-1,1$ [ such that

$$
\left[\begin{array}{cccc}
-\gamma^{2} I & * & * & * \\
B_{i}^{w} & \alpha^{2} S_{i}-S_{j} & * & A_{i} G_{i}+\alpha_{i} G_{i}-\alpha_{i} S_{i} \\
D_{i}^{z w} & 0 & -I & C_{i}^{z} G \\
0 & * & * & S_{i}-\left(G_{i}+G_{i}^{T}\right)
\end{array}\right]<0 .
$$

Proof. It follows much the same way as above for the first steps leading to

$$
\begin{aligned}
& {\left[\begin{array}{cccc}
-S_{i} & * & * & * \\
0 & -\gamma^{2} I & * & * \\
A_{i} S_{i} & B_{i}^{w} & -S_{j} & * \\
C_{i}^{z} S_{i} & D_{i}^{z w} & 0 & -I
\end{array}\right]} \\
& =\underbrace{\left[\begin{array}{cccc}
-S_{i} & * & * & * \\
0 & -\gamma^{2} I & * & * \\
-\alpha_{i} S_{i} & B_{i}^{w} & -S_{j} & * \\
0 & D_{i}^{z w} & 0 & -I
\end{array}\right]}_{\Phi}+\underbrace{\left[\begin{array}{c}
0 \\
0 \\
A_{i}+\alpha_{i} I \\
C_{i}^{z}
\end{array}\right]}_{N} \underbrace{\left[\begin{array}{llll}
S_{i} & 0 & 0 & 0
\end{array}\right]}_{J^{T}} \\
& +\underbrace{\left[\begin{array}{c}
S_{i} \\
0 \\
0 \\
0
\end{array}\right]}_{J} \underbrace{\left[\begin{array}{llll}
0 & 0 & A_{i}+\alpha_{i} I & C_{i}^{z}
\end{array}\right]}_{N}<0
\end{aligned}
$$

which is transformed using Lemma 1 and Schur complement technique.

Of course, with $\alpha_{i}=0$ is recovered the result given in [21].

\section{Main Result}

This section gives the main results of the paper. First, based on the switched quadratic Lyapunov function and Projection Lemma, new sufficient LMI conditions are deduced to obtain stabilizing SOF controller gains. Then, the method is extended to SOF controller design with $\gamma$-performance.

4.1. Static Output Feedback Control. Given the measurements

$$
y(k)=C_{i} x(k)
$$

our main result consists on the determination of a switched static output feedback controller:

$$
u(k)=K_{i} y(k)
$$

such that the closed loop system is asymptotically stable. To this end, let us introduce the closed loop switched system:

$$
x(k+1)=\left(A_{i}+B_{i} K_{i} C_{i}\right) x(k) .
$$

The problem is then reduces to find $P_{i}$ and $K_{i}$ (for all $i \in \xi$ ), such that

$$
\left[\begin{array}{cc}
-P_{i} & \left(A_{i}+B_{i} K_{i} C_{i}\right)^{T} P_{j} \\
P_{j}\left(A_{i}+B_{i} K_{i} C_{i}\right) & -P_{j}
\end{array}\right]<0
$$

for all $(i, j) \in \xi \times \xi$, or equivalently to find $S_{i}, G_{i}$, and $K_{i}$ such that

$$
\left[\begin{array}{cc}
-G_{i}-G_{i}^{T}+S_{i} & G_{i}^{T}\left(A_{i}+B_{i} K_{i} C_{i}\right)^{T} \\
\left(A_{i}+B_{i} K_{i} C_{i}\right) G_{i} & -S_{j}
\end{array}\right]<0
$$

for all $(i, j) \in \xi \times \xi$, or equivalently to find $S_{i}, G_{i}, K_{i}$, and $\alpha_{i}$ which are arbitrary prescribed numbers in ] - 1, 1[ such that

$$
\left[\begin{array}{cc}
\alpha_{i}^{2} S_{i}-S_{j} & \mathcal{A} G_{i}+\alpha_{i} G_{i}-\alpha_{i} S_{i} \\
G_{i}^{T} \mathcal{A}^{T}+\alpha_{i} G_{i}^{T}-\alpha_{i} S_{i} & S_{i}-G_{i}-G_{i}^{T}
\end{array}\right]<0,
$$

where $\mathcal{A}$ denotes $\left(A_{i}+B_{i} K_{i} C_{i}\right)$ for all $(i, j) \in \xi \times \xi$. The problem of solving (24), (25), or (26) for $\left(P_{i}, K_{i}\right),\left(S_{i}, G_{i}, K_{i}\right)$ or $\left(S_{i}, G_{i}, K_{i}, \alpha_{i}\right)$, respectively, is nonconvex in general. This makes the problem of output feedback a very difficult one. A sufficient condition is given in the following theorems. These conditions have the advantage of being convex and to be numerically tractable.

Theorem 4. If there exist symmetric matrices $S_{i}$, matrices $U_{i}$ and $V_{i}($ for all $i \in \xi)$ such that for all $(i, j) \in \xi \times \xi$ [14]

$$
\begin{gathered}
{\left[\begin{array}{cc}
-S_{i} & \left(A_{i} S_{i}+B_{i} U_{i} C_{i}\right)^{T} \\
A_{i} S_{i}+B_{i} U_{i} C_{i} & -S_{j}
\end{array}\right]<0,} \\
V_{i} C_{i}=C_{i} S_{i}, \quad \forall i \in \xi,
\end{gathered}
$$

then the output feedback given by (22) with

$$
K_{i}=U_{i} V_{i}^{-1}, \quad \forall i \in \xi
$$

asymptotically stabilizes (1). 
Remarks. (i) The matrix $C=U\left[C_{0} 0\right] V^{T}$ is the singular value decomposition of matrix $C, V=\left[\begin{array}{ll}V_{1} & V_{2}\end{array}\right]$ and $S$ having the following particular structure: $S=V\left[\begin{array}{cc}S_{11} & 0 \\ 0 & S_{22}\end{array}\right] V^{T}$.

Proof (see [14]). Assume that there exist $S_{i}, U_{i}$, and $V_{i}$ such that (27) and (28) are satisfied. As $C_{i}$ is of full-row rank and $S_{i}$ is positive definite, it follows from (28) that $V_{i}$ is of full rank for all $i=1, \ldots, N$ and then invertible. From (28) and (29), we get $U_{i} C_{i}=K_{i} C_{i} S_{i}$, for all $i \in \xi$.

Replacing $U_{i} C_{i}$ in (27) by $K_{i} C_{i} S_{i}$ and applying the Schur complement formula one gets for all $(i, j) \in \xi \times \xi$

$$
\left(A_{i}+B_{i} K_{i} C_{i}\right)^{T} S_{j}^{-1}\left(A_{i}+B_{i} K_{i} C_{i}\right)-S_{i}^{-1}<0 .
$$

Letting $P_{i}=S_{i}^{-1}$ and $P_{j}=S_{j}^{-1}$ and using the Schur complement formula, the latest inequality is nothing than the stability condition (10) applied to the closed-loop system (23). Hence, by Theorem 2, the closed loop system is asymptotically stable.

Theorem 5. If there exist symmetric matrices $S_{i}$, matrices $G_{i}$, $U_{i}$ and $V_{i}$ (for all $\left.i \in \xi\right)$ such that for all $(i, j) \in \xi \times \xi$ [14]

$$
\begin{gathered}
{\left[\begin{array}{cc}
-G_{i}-G_{i}^{T}+S_{i} & \left(A_{i} G_{i}+B_{i} U_{i} C_{i}\right)^{T} \\
A_{i} G_{i}+B_{i} U_{i} C_{i} & -S_{j}
\end{array}\right]<0,} \\
V_{i} C_{i}=C_{i} G_{i}, \quad \forall i \in \xi,
\end{gathered}
$$

then the output feedback control given by (22) with

$$
K_{i}=U_{i} V_{i}^{-1}, \quad \forall i \in \xi
$$

stabilizes the system (1).

Remarks. (i) The matrix $C=U\left[\begin{array}{ll}C_{0} & 0\end{array}\right] V^{T}$ is the singular value decomposition of matrix $C, V=\left[\begin{array}{ll}V_{1} & V_{2}\end{array}\right]$, the matrix $S$ having a general structure and $G$ having the following particular form:

$$
G=V\left[\begin{array}{cc}
G_{11} & 0 \\
G_{21} & G_{22}
\end{array}\right] V^{T} .
$$

Proof (see [14]). First, notice that if (31) holds, then $-G_{i}-$ $G_{i}^{T}+S_{i}<0$ and the matrices $G_{i}$ are full rank. Hence, matrices $V_{i}$ satisfying (32) are nonsingular. Following similar arguments as in the proof of Theorem 4 we find that satisfying conditions of Theorem 5 leads to for all $(i, j) \in$ $\xi \times \xi$

$$
\left[\begin{array}{cc}
-G_{i}-G_{i}^{T}+S_{i} & G_{i}^{T}\left(A_{i}+B_{i} K_{i} C_{i}\right)^{T} \\
\left(A_{i}+B_{i} K_{i} C_{i}\right) G_{i} & -S_{j}
\end{array}\right]<0,
$$

which is nothing than (11) applied to the closed system (23). Hence, by Theorem 2, the closed-loop system is asymptotically stable.
Theorem 6. If there exist symmetric matrices $S_{i}$, matrices $G_{i}, U_{i}, V_{i}$, and $\alpha_{i}$ which are arbitrary prescribed numbers in ] - 1,1 [ (for all $i \in I)$ such that for all $(i, j) \in \xi \times \xi$

$$
\left[\begin{array}{cc}
\alpha_{i}^{2} S_{i}-S_{j} & \mathcal{B}+\alpha_{i} G_{i}-\alpha_{i} S_{i} \\
\mathcal{B}^{T}+\alpha_{i} G_{i}^{T}-\alpha_{i} S_{i} & S_{i}-G_{i}-G_{i}^{T}
\end{array}\right]<0,
$$

where $B$ denotes $A_{i} G_{i}+B_{i} U_{i} C_{i}$, and

$$
V_{i} C_{i}=C_{i} G_{i}, \quad \forall i \in \xi,
$$

then the output feedback control given by (22) with

$$
K_{i}=U_{i} V_{i}^{-1}, \quad \forall i \in \xi
$$

stabilizes the system (1).

Proof. First, notice that if (36) holds, then $-G_{i}-G_{i}^{T}+S_{i}<$ 0 and the matrices $G_{i}$ are full rank. Hence, matrices $V_{i}$ satisfying (37) are nonsingular. Following similar arguments as in the proof of Theorem 5 [14] we find that satisfying conditions of Theorem 6 leads to for all $(i, j) \in \xi \times \xi$

$$
\left[\begin{array}{cc}
\alpha_{i}^{2} S_{i}-S_{j} & \mathcal{B}+\alpha_{i} G_{i}-\alpha_{i} S_{i} \\
\mathcal{B}^{T}+\alpha_{i} G_{i}^{T}-\alpha_{i} S_{i} & S_{i}-G_{i}-G_{i}^{T} \mathrm{~d}
\end{array}\right]<0,
$$

which is nothing than (12) applied to the closed system (23). Hence, by Theorem 2, the closed-loop system is asymptotically stable.

4.2. Static Output Feedback Control with $\gamma$-Performance. This section gives control design result that directly comes from the preceding section so that the proof will be almost omitted. Let now the linear switched system be controlled described by

$$
\begin{aligned}
x(k+1) & =A_{i} x(k)+B_{i} u(k)+B_{i}^{w} w(k), \\
z(k) & =C_{i}^{z} x(k)+D_{i}^{z} u(k)+D_{i}^{z w} w(k), \\
y(k) & =C_{i} x(k)+D_{i}^{y w} w(k),
\end{aligned}
$$

where $x(k) \in I R^{n}$ is the state vector, $u(k) \in I R^{m}$ the control input, $w(k) \in I R^{q}$ the disturbance, $z(k) \in I R^{p}$ the controlled output vector, and $y(k) \in I R^{r}$ the measured output.

We are interested by the design of a switched static output feedback control given by (22) such that the closed loop system

$$
\begin{aligned}
x(k+1) & =\left(A_{i}+B_{i} K_{i} C_{i}\right) x(k)+B_{i}^{w} w(k), \\
z(k) & =\left(C_{i}^{z}+D_{i}^{z} K_{i} C_{i}\right) x(k)+D_{i}^{z w} w(k)
\end{aligned}
$$

is asymptotically stable and satisfies the $\gamma$-performance. The following theorem gives sufficient condition to build the switched SOF control (22). 
Theorem 7. If for all $(i, j) \in \xi \times \xi$, there exist symmetric matrices $S_{i}$, matrices $G_{i}$, and scalar variables $\left.\alpha_{i} \in\right]-1,1[$ solution of the following LMI:

$$
\left[\begin{array}{cccc}
-\gamma^{2} I & * & * & * \\
B_{i}^{w} & \alpha_{i}^{2} S_{i}-S_{j} & * & A_{i} G_{i}+B_{i} U_{i} C_{i}+\alpha_{i} G_{i}-\alpha_{i} S_{i} \\
D_{i}^{z w} & 0 & -I & C_{i}^{z} G_{i}+D_{i}^{z} U_{i} C_{i} \\
0 & * & * & S_{i}-G_{i}-G_{i}^{T}
\end{array}\right]<0,
$$

then the closed-loop system (41) is asymptotically stable and the switched SOF controller

$$
K_{i}=U_{i} V_{i}^{-1}, \quad \forall i \in \xi
$$

ensures the $\gamma$-performance for the closed-loop system.

\section{Numerical Evaluation}

In this section, a numerical evaluation is proposed. The problem considered here is the design of a static output feedback controller stabilizing the switched system. The result obtained using the Theorem 6 is compared to the three methods developed in [14] and summarized in the Table 1. The switched system is characterized by the number of modes $(N)$, the system order $(n)$, number of inputs $(m)$, and the number of outputs $(p)$. For fixed values of $(N, n, m, p)$, we generate randomly 100 switched systems of the form (1). So the purpose is to design by using four methods a feedback controller in the form (18) such that the closed-loop system is stable.

(i) Method1 uses constant Lyapunov function (CLF) $V\left(x_{k}\right)=x_{k}^{T} P x_{k}$.

This corresponds to conditions in Theorem 4 with for all $(i, j) \in \xi \times \xi, S_{i}=S_{j}=S$ a constant matrix.

(ii) Method2 uses the conditions given in Theorem 4.

(iii) Method3 uses the conditions given in Theorem 5.

(iv) Method4 uses the conditions given in Theorem 6.

By using the matlab LMI Control Toolbox to check the feasibility of the LMI conditions, we introduce a counter (Success Method1, Success Method2, Success Method3, and Success method4) which is increased if the corresponding method succeeds in providing an output feedback stabilizing control.

\section{Illustrative Example}

The simple illustrative switched system is constituted of two subsystems with different dynamic, control, and output matrices. The data are $(m=1)$ and the number of outputs ( $p$ $=1$ ).

The proposed approach improves, in terms of norm

\begin{tabular}{|c|c|c|}
\hline Switched system & Success & $N=2$ \\
\hline$n=3$ & Method1 & 76 \\
\hline$m=1$ & Method2 & 83 \\
\hline \multirow[t]{2}{*}{$p=1$} & Method3 & 88 \\
\hline & Method4 & 95 \\
\hline$n=4$ & Method1 & 40 \\
\hline$m=1$ & Method2 & 63 \\
\hline \multirow[t]{2}{*}{$p=1$} & Method3 & 67 \\
\hline & Method4 & 87 \\
\hline$n=5$ & Method1 & 8 \\
\hline$m=1$ & Method2 & 20 \\
\hline \multirow[t]{2}{*}{$p=1$} & Method3 & 32 \\
\hline & Method4 & 52 \\
\hline$n=5$ & Method1 & 61 \\
\hline$m=2$ & Method2 & 87 \\
\hline \multirow[t]{2}{*}{$p=2$} & Method3 & 93 \\
\hline & Method4 & 97 \\
\hline$n=4$ & Method1 & 66 \\
\hline$m=2$ & Method2 & 81 \\
\hline \multirow{2}{*}{$p=1$} & Method3 & 82 \\
\hline & Method4 & 100 \\
\hline$n=5$ & Method1 & 35 \\
\hline$m=2$ & Method2 & 65 \\
\hline \multirow[t]{2}{*}{$p=1$} & Method3 & 68 \\
\hline & Method4 & 92 \\
\hline$n=4$ & Method1 & 66 \\
\hline$m=1$ & Method2 & 73 \\
\hline \multirow[t]{2}{*}{$p=2$} & Method3 & 85 \\
\hline & Method4 & 90 \\
\hline$n=5$ & Method1 & 20 \\
\hline$m=1$ & Method2 & 42 \\
\hline \multirow[t]{2}{*}{$p=2$} & Method3 & 58 \\
\hline & Method4 & 73 \\
\hline$n=7$ & Method1 & 0 \\
\hline$m=2$ & Method2 & 16 \\
\hline \multirow[t]{2}{*}{$p=1$} & Method3 & 25 \\
\hline & Method4 & 30 \\
\hline
\end{tabular}
bound, the results given in [14].
TABLE 1: Numerical evaluation.

The contribution of this paper stands in the combination of poly-quadratic stability concept [14] with slack $G_{i}$ matrices and "slack" scalars $\alpha_{i}$, with ad hoc changes of variables to provide improved LMI conditions that enables the design of a switched static output-feedback.

The Tables 2 and 3 improve in terms of norm bound the results given in [16].

\section{Summary and Conclusions}

In this paper, the problem of stability and synthesis of discrete switched linear systems using switched Lyapunov function (SLF) is investigated. Using these Lyapunov function, the problem of stability analysis and the static output 
TABLE 2: Numerical example.

\begin{tabular}{lc}
\hline Mode1 & Mode2 \\
\hline$A_{1}=\left[\begin{array}{ll}0.2 & 0.1 \\
0.6 & 0.3\end{array}\right]$ & $A_{2}=\left[\begin{array}{ll}0.1 & 0.2 \\
0.3 & 0.4\end{array}\right]$ \\
$B_{1}=\left[\begin{array}{l}0.34 \\
-0.3\end{array}\right]$ & $B_{2}=\left[\begin{array}{l}0.1 \\
-1\end{array}\right]$ \\
$B_{1}^{w}=\left[\begin{array}{ll}1.6 & 0.1 \\
2.30 .2\end{array}\right]$ & $B_{2}^{w}=\left[\begin{array}{ll}1.2 & 0.7 \\
1.1 & 0.2\end{array}\right]$ \\
$C_{1}^{z}=\left[\begin{array}{ll}0.15 & 0.3\end{array}\right]$ & $C_{2}^{z}=\left[\begin{array}{ll}-0.19 & 0.17\end{array}\right]$ \\
$D_{1}^{z}=1.2$ & $D_{2}^{z}=0.2$ \\
$D_{1}^{z w}=\left[\begin{array}{ll}0.03 & 0.2\end{array}\right]$ & $D_{2}^{z w}=\left[\begin{array}{ll}0.02 & 0.1\end{array}\right]$ \\
$C_{1}=\left[\begin{array}{ll}0.29 & 0.15\end{array}\right]$ & $C_{2}=\left[\begin{array}{ll}-0.19 & 0.17\end{array}\right]$ \\
$D_{1}^{y w}=\left[\begin{array}{ll}0.03 & 0.2\end{array}\right]$ & $D_{2}^{y w}=\left[\begin{array}{ll}0.30 .2\end{array}\right]$ \\
\hline
\end{tabular}

TABLE 3: Numerical results.

\begin{tabular}{lcc}
\hline Approach & Controller gains & Upper bounds $\gamma$ \\
\hline $\begin{array}{l}\text { Theorem } 4 \text { with } \\
\gamma \text {-performance }\end{array}$ & $\begin{array}{l}K_{\text {model }}=-0.9542 ; \\
K_{\text {mode2 }}=-1.4861\end{array}$ & 0.96 \\
\hline Approach [14] & $\begin{array}{l}K_{\text {model }}=-0.9176 ; \\
K_{\text {mode2 }}=-1.5775\end{array}$ & 0.58 \\
\hline New approach, $\alpha=0.5$ & $\begin{array}{l}K_{\text {model }}=-0.9368 ; \\
K_{\text {mode2 }}=-1.7066\end{array}$ & 0.54 \\
\hline
\end{tabular}

feedback design have been studied. Based on the stability of polytopic time-varying uncertain systems, condition for stability analysis of switched systems has been proposed. The existence of an SLF to ensure stability of a discrete switched system is proven to be equivalent to three-LMIbased conditions considered in [14]. The derived conditions are expressed as a family of linear matrix inequalities (LMIs) parameterized by the scalar variables $\alpha_{i}$. These conditions reduce significantly the conservatism and show the advantage of using the scalar variables in the case of static output feedback. Numerical evaluations are given to demonstrate the applicability and the conservatism reduction of the proposed conditions and a comparison with recent conditions proposed in literature has been described.

\section{References}

[1] S. M. Williams and R. G. Hoft, "Adaptive frequency domain control of PPM switched power line conditioner," IEEE Transactions on Power Electronics, vol. 6, pp. 665-670, 1991.

[2] D. Liberzon and A. S. Morse, "Basic problems in stability and design of switched systems," IEEE Control Systems Magazine, vol. 19, no. 5, pp. 59-70, 1999.

[3] A. S. Morse, "Supervisory control of families of linear setpoint controllers-part 1: exact matching," IEEE Transactions on Automatic Control, vol. 41, no. 10, pp. 1413-1431, 1996.

[4] M. Zefrani and J. W. Burdick, "Design of switching controllers for systems with changing dynamics," in Proceedings of the 37th IEEE Conference on Decision and Control, pp. 2113-2118, Tampa, Fla, USA, December 1998.

[5] F. A. Cuzzola and M. A. Morari, "A generalized approach for analysis and control of discrete-time piecewise affine and hybrid systems," in Hybrid Systems: Computation and Control, Springer, Berlin, Germany, 2001.
[6] D. Liberzon, J. P. Hespanha, and A. S. Morse, "Stability of switched system: a Lie-algebraic condition," Systems and Control Letters, vol. 37, pp. 117-122, 1999.

[7] H. Ye, A. N. Michel, and L. Hou, "Stability theory for hybrid dynamical systems," in Proceedings of the 34th IEEE Conference on Decision and Control, vol. 3, pp. 2679-2684, New Orleans, La, USA, December 1995.

[8] G. I. Bara and M. Boutyeb, "Switched output feedback stabilization of discrete-time switched systems," in Proceedings of the 45th IEEE Conference on Decision and Control, San Diego, Calif, USA, December 2006.

[9] G. I. Bara, "Robust switched output feedback control for discrete-time switched linear systems," in Proceedings of the 46th IEEE Conference on Decision and Control, pp. 4986-4992, New Orleans, La, USA, December 2007.

[10] D.-W. Ding and G.-H. Yang, "Static output feedback control for discrete-time switched linear systems under arbitrary switching," in Proceedings of the American Control Conference, Staint Louis, Mo, USA, June 2009.

[11] X. D. Koutsoukos and P. J. Antsaklis, "Design of stabilizing switching control laws for discrete- and continuous-time linear systems using piecewise-linear Lyapunov functions," International Journal of Control, vol. 75, no. 12, pp. 932-945, 2002.

[12] R. A. DeCarlo, M. Branicky, S. Pettersson, and B. Lennartson, "Perspectives and results on the stability and stabilizability of hybrid systems," Proceedings of the IEEE, vol. 88, no. 7, pp. 1069-1082, 2000.

[13] M. S. Branicky, "Multiple Lyapunov functions and other analysis tools for switched and hybrid systems," IEEE Transactions on Automatic Control, vol. 43, no. 4, pp. 475-482, 1998.

[14] J. Daafouz, P. Riedinger, and C. Iung, "Stability analysis control synthesis for switched systems:a switched Lyapunov function approach," IEEE Transactions on Automatic Control, vol. 47, no. 11, pp. 1883-1887, 2002.

[15] J. Daafouz and J. Bernussou, "Parameter dependent Lyapunov functions for discrete time systems with time varying parametric uncertainties," Systems and Control Letters, vol. 43, no. 5, pp. 355-359, 2001.

[16] J. C. Geromel and P. Colaneri, "Stability and stabilization of discrete time switched systems," International Journal of Control, vol. 79, no. 7, pp. 719-728, 2006.

[17] J. C. Geromel, C. C. de Souza, and R. E. Skelton, "Static output feedback controllers: stability and convexity," IEEE Transactions on Automatic Control, vol. 43, no. 1, pp. 120-125, 1998.

[18] J. Dong and G.-H. Yang, "Static output feedback control synthesis for linear systems with timeinvariant parametric uncertainties," IEEE Transactions on Automatic Control, vol. 52, no. 10, pp. 1930-1936, 2007.

[19] S. Boyd, L. El Ghaoui, E. Feron, and V. Balakrishnan, Linear Matrix Inequality in System and Control Theory, vol. 15 of SIAM Studies in Applied Mathematics, SIAM, Philadelphia, Pa, USA, 1994.

[20] M. Vidyasagar, Nonlinear Systems Analysis, Prentice-Hall, Upper Saddle River, NJ, USA, 1993.

[21] J. Daafouz and J. Bernussou, "Robust dynamic output feedback control for switched systems," in Proceedings of the 41st IEEE Conference on Decision and Control, vol. 4, pp. 43894394, Las Vegas, Nev, USA, December 2002. 

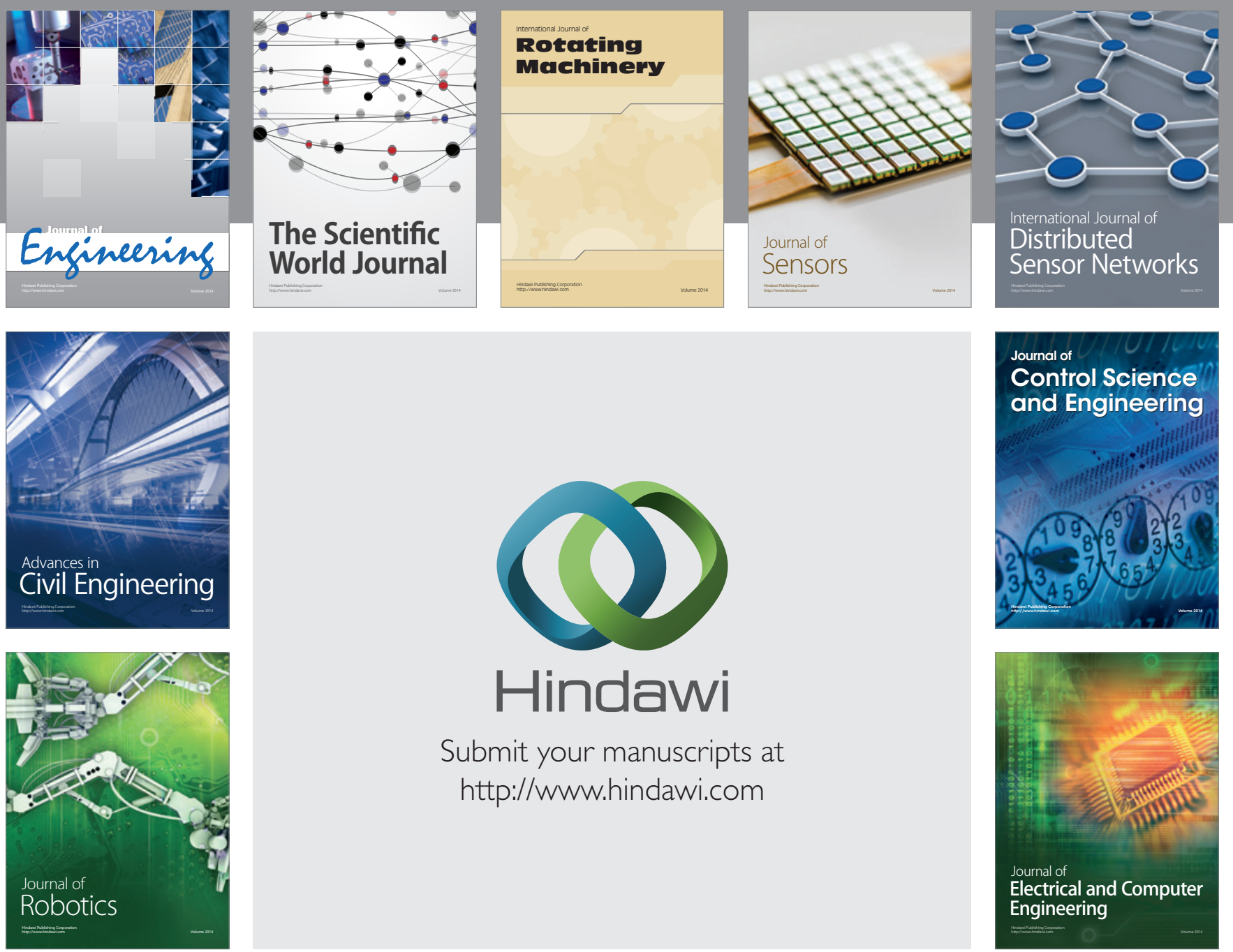

Submit your manuscripts at

http://www.hindawi.com
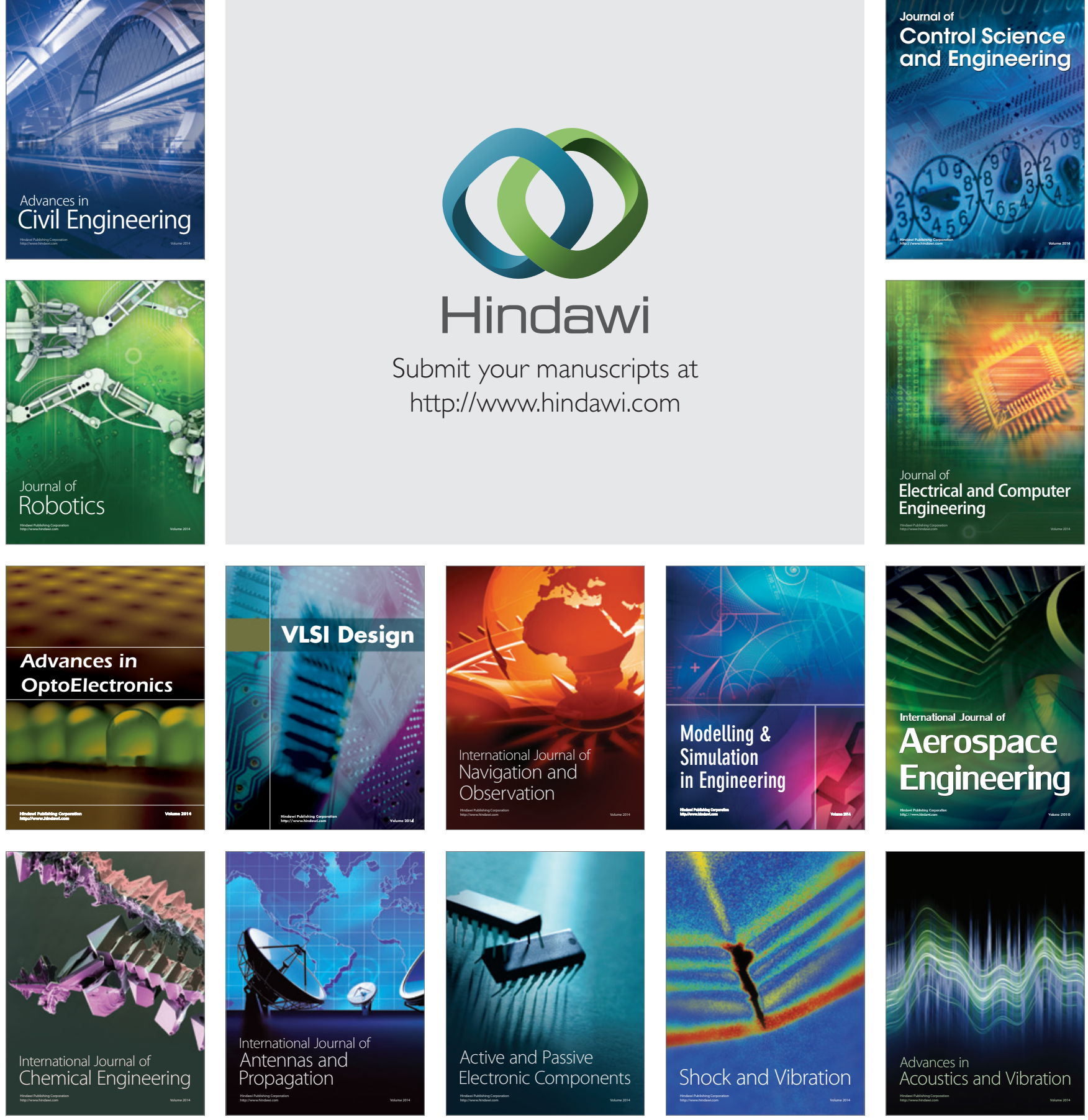